\title{
Crossing the Resolution Limit in Near-Infrared Imaging of Silicon Chips: Targeting 10-nm Node Technology
}

\author{
Krishna Agarwal, ${ }^{1, *}$ Rui Chen, ${ }^{2}$ Lian Ser Koh, ${ }^{3}$ Colin J. R. Sheppard, ${ }^{4}$ and Xudong Chen ${ }^{2}$ \\ ${ }^{1}$ Singapore-MIT Alliance for Research and Technology (SMART) Centre, CREATE Tower, \\ Singapore 138602 \\ ${ }^{2}$ Department of Electrical and Computer Engineering, National University of Singapore, \\ Singapore 117583 \\ ${ }^{3}$ Semicaps Private Limited, Singapore 139959 \\ ${ }^{4}$ Istituto Italiano di Tecnologia, Via Morego 30, 16163 Genoa, Italy \\ (Received 9 June 2014; revised manuscript received 3 December 2014; published 6 May 2015)
}

\begin{abstract}
The best reported resolution in optical failure analysis of silicon chips is 120-nm half pitch demonstrated by Semicaps Private Limited, whereas the current and future industry requirement for 10-nm node technology is 100-nm half pitch. We show the first experimental evidence for resolution of features with 100 -nm half pitch buried in silicon $(\lambda / 10.6)$, thus fulfilling the industry requirement. These results are obtained using near-infrared reflection-mode imaging using a solid immersion lens. The key novel feature of our approach is the choice of an appropriately sized collection pinhole. Although it is usually understood that, in general, resolution is improved by using the smallest pinhole consistent with an adequate signal level, it is found that in practice for silicon chips there is an optimum pinhole size, determined by the generation of induced currents in the sample. In failure analysis of silicon chips, nondestructive imaging is important to avoid disturbing the functionality of integrated circuits. High-resolution imaging techniques like SEM or TEM require the transistors to be exposed destructively. Optical microscopy techniques may be used, but silicon is opaque in the visible spectrum, mandating the use of near-infrared light and thus poor resolution in conventional optical microscopy. We expect our result to change the way semiconductor failure analysis is performed.
\end{abstract}

DOI: 10.1103/PhysRevX.5.021014

\section{INTRODUCTION}

Imaging silicon integrated circuits (ICs) nondestructively is important for failure localization and analysis, which in turn helps in yield enhancement. With miniaturization of ICs, the resolution demands for nondestructive imaging techniques of silicon ICs have been consistently increasing, requiring better and better resolution for failure analysis (FA) and yield enhancement purposes. International Technology Roadmap for Semiconductors (ITRS) [1], the annually generated road map that sets requirements and expectations for the semiconductor industry, states that a half-pitch resolution of $100 \mathrm{~nm}$ is needed for FA of flash memory of half-pitch 16-12 nm (currently the state of the art). It also states that technology solutions that can achieve

\footnotetext{
*Corresponding author. uthkrishth@gmail.com

This author was affiliated with the Department of Electrical and Computer Engineering, National University of Singapore when conducting the work.
}

Published by the American Physical Society under the terms of the Creative Commons Attribution 3.0 License. Further distribution of this work must maintain attribution to the author(s) and the published article's title, journal citation, and DOI.
Subject Areas: Electronics, Optics

this target are unavailable. This report further indicates that one of the difficult challenges in the time frames of 2013-2020 and beyond 2020 is the development of an optical scanning microscopy technique that can help in localization of physical defects such that the test cycle time can be reduced significantly. The reason is twofold. Firstly, physical localization of the defects early in the fabrication cycle allows for correction or improvement of fabrication masks and processes before mass fabrication and thus allows for throughput improvement. Secondly, localization of the defects reduces the automatic test cycles of the dies since the complicated functional analysis can be focused on the problem areas directly and need not either select random locations for functional analysis or perform testing on the entire chip with millions of transistors. Thus, a high-resolution optical imaging technique is of critical importance to the semiconductor industry and the industry anxiously awaits a good imaging solution that can image large areas quickly and yet provide node-level high-resolution image.

A silicon chip can be imaged through the top plane (front side) or the bottom plane (back side). Imaging transistors through the top plane is difficult because transistors are buried beneath several metallization and circuital layers [see Fig. 1(a)]. Although such layers are absent towards the bottom plane, transistors either need to be exposed 


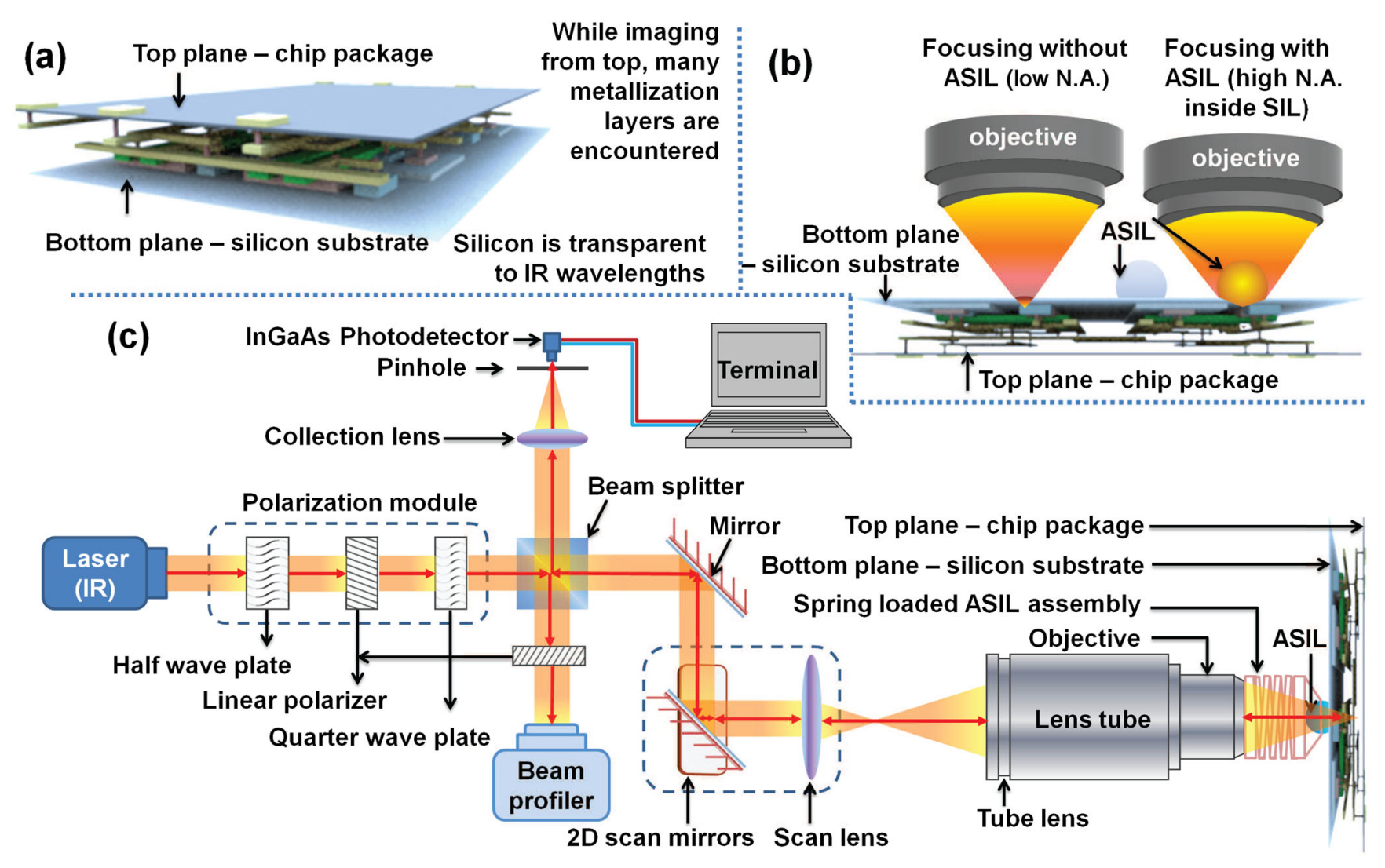

FIG. 1. Use of ASIL for imaging integrated circuits. Imaging through the top surface is difficult due to the presence of many metallization layers, seen in yellow in (a). On the other hand, imaging through the bottom plane is feasible since the transistor structures are typically in the first layer from the bottom and the silicon substrate is transparent to near-infrared waves (a). As opposed to imaging using only an objective lens, using an additional aplanatic solid immersion lens increases the effective N.A. inside the SIL, thus providing better resolution (b). The experimental setup of the ASIL scanning microscope is shown in (c).

destructively for high-resolution imaging techniques like SEM or TEM, or the light used for imaging should be able to penetrate the substrate. Near-infrared (NIR) light used in reflection-mode microscopy meets the criteria because silicon is transparent to NIR wavelengths $(1-2 \mu \mathrm{m})$ and the reflected path does not encounter other circuital layers when imaging is done through the bottom plane. Other optical wavelengths cannot be used since silicon is opaque to visible and ultraviolet radiation. Nevertheless, the long wavelengths of the NIR range (1-2 $\mu \mathrm{m})$ make the Rayleigh resolution limit of approximately half wavelength impractically large. Yet, NIR still holds promise thanks to the pioneering research on a solid immersion lens (SIL) made of silicon [2], which predicts resolution enhancement by a factor of at least $n$, the refractive index of silicon, over the Rayleigh resolution limit of about a half wavelength [3].

In SIL technology, a spherical lens made of silicon and sliced at an appropriate plane is pressed onto the silicon substrate such that the resolution of the system can be enhanced. Notably, two designs of SIL provide an aberration-free focal spot, namely, the hemispherical SIL and the aplanatic solid immersion lens (ASIL). An ASIL provides an additional advantage of increased N.A. [see Fig. 1(b)] and gives a lateral magnification of $n_{\mathrm{SIL}}^{2}$ as compared to the lateral magnification of $n_{\mathrm{SIL}}$ in the hemispherical SIL [3]. An ASIL also avoids introduction of coma in the imaging of extended objects.

Despite the salient features of NIR and SIL imaging technology, enhancing the resolution of NIR-SIL reflection-mode microscopy is an ongoing endeavor. Initially, Refs. [4,5] measured the FWHM of the intensity profile when a line feature is scanned as an indicator of resolution. The FWHM reported by them is $230 \mathrm{~nm}$, achieved using $\lambda=1200 \mathrm{~nm}$. This corresponds to $0.19 \lambda$ or $192 \mathrm{~nm}$ for the shortest possible wavelength of $1 \mu \mathrm{m}$. Semicaps Pte. Ltd. demonstrated a more practically useful resolution approach in 2012 [6] by showing that the lines in a big feature with multiple lines, having a half pitch of $120 \mathrm{~nm}$, can be resolved using 1064-nm wavelength. The superior resolution reported in Ref. [6] as compared to previous observations has been attributed to the enhancement of the physically available N.A. inside silicon N.A.SIL to 3.3.

Ongoing and recent works on theoretical analysis [7-12] and computational modeling [13-17] of ASIL microscopy have paved the path for performance improvement of NIR-ASIL reflection-mode microscopy for silicon FA. It was shown computationally in Refs. [13,18] that a 
resolution of $\approx 0.09 \lambda$ (i.e., $96-98 \mathrm{~nm}$ for $\lambda=1064 \mathrm{~nm}$ ) should be possible using appropriate combinations of polarization, filter, and radius of pinhole.

Here, we present the first experimental evidence of resolving features of $100-\mathrm{nm}$ half pitch in a silicon substrate imaged through the bottom plane using NIRSIL reflection-mode microscopy. We use the computational results and system design of Chen et al. [13,15] to obtain the presented experimental results. Our resolution of $100 \mathrm{~nm}$ for NIR-SIL imaging is 20 -nm improved over the industrially demonstrated best resolution of 120-nm half pitch [6]. Our result shows that the resolution required for nondestructive analysis of current and future semiconductor technologies can be achieved practically. Following this result, we believe that NIR-ASIL reflection-mode microscopy can meet the FA demands of the semiconductor industry for years to come and satisfy the requirements for 10-nm node technology, changing the way FA is done.

A major contribution of our work is to break the conventional and well-accepted notion that a smaller pinhole in a scanning system always translates to better image and better resolution. It is shown in this paper that for the ASIL scanning mode microscope considered here, a pinhole of $17.5 \mu \mathrm{m}$ gives better imaging results in comparison to 12.5 and $5 \mu \mathrm{m}$. We also show that it is mainly due to the effect of the longitudinal current on the image in a high N.A. system such as an ASIL microscope. Here, we should point out that this work is critically different from Refs. $[13,15]$. In Ref. [13], cylindrical vector beams were considered, among which azimuthal and azimuthal vortex beams are completely devoid of longitudinal focal fields while a radially polarized beam is known to promote a more predominant role for longitudinal focal fields. In either case, longitudinal currents-their complete absence or predominance - are the defining characteristics, irrespective of the N.A. of the system. On the contrary, longitudinal currents gain importance for noncylindrical vector polarizations, such as circular polarization, in the case of high N.A. systems only, and the effect of longitudinal currents for such situations has seldom been studied. To our knowledge, no other publication, including Ref. [13], discusses explicitly how the longitudinal currents affect high N.A. systems that employ noncylindrical vector polarizations and how such longitudinal currents should influence system design such as pinhole dimension selection. This paper intends to fill this gap. The results in Ref. [15] did consider 3 pinhole radii, but their dimensions were not chosen on the basis of longitudinal currents. They were rather chosen based on confocal, Airy-disk size, and wide-field setups, and the choice was used to present some qualitative differences between wide-field, typical scanning (with pinhole of Airy-disk size) and confocal modes of the system. It is only in this paper that the design of pinhole dimension for a circular polarized beam, which does not require a specialized complicated setup and extensive alignment procedures, is considered and shown to deliver a resolution beyond the current state of the art. We note that while the role of the pinhole size is examined for silicon chips here, the work also indicates that the pinhole selection can improve the resolution for other high N.A. imaging systems, such as is used in bioimaging applications.

\section{RESULTS}

In our system, we use 1064-nm wavelength and a NIR-SIL system with N.A.SIL $=3.3$ (determined by the SIL assembly). The microscopy setup used in our experiment is shown in Fig. 1(c). We use circular polarization and pinholes of different radii. A SIL assembly [19] is used to hold the SIL, which also helps to avoid an air gap between the sample and SIL, and to accurately locate the focal plane of ASIL. We note that this assembly plays an important role since the SIL system is quite prone to aberrations [8,11,20-22]. This assembly allows an effective numerical aperture N.A.SIL of 3.3. TedPella Inc.'s critical dimension calibration or resolution test target is used as the sample [23]. It is a silicon chip with features etched upon its top surface. The central region of the sample has three critical dimension features with pitches 500, 200, and $100 \mathrm{~nm}$ and corresponding half pitches of 250,100, and $50 \mathrm{~nm}$, which are shown in Fig. 2(a). Among them, the second feature is our feature of interest, since it matches the resolution requirement set by ITRS.

The image of the sample obtained using our system and pinhole of radius $17.5 \mu \mathrm{m}$ is shown in Fig. 2(b) and its zoom-in around the second feature is shown in Fig. 2(d). The intensity across the cross-section line shown in Fig. 2(d) is plotted above it. For convenience of visualization, a pseudocolored image of the feature is shown in Fig. 2(c). It is seen from Figs. 2(c) and 2(d) that all five lines

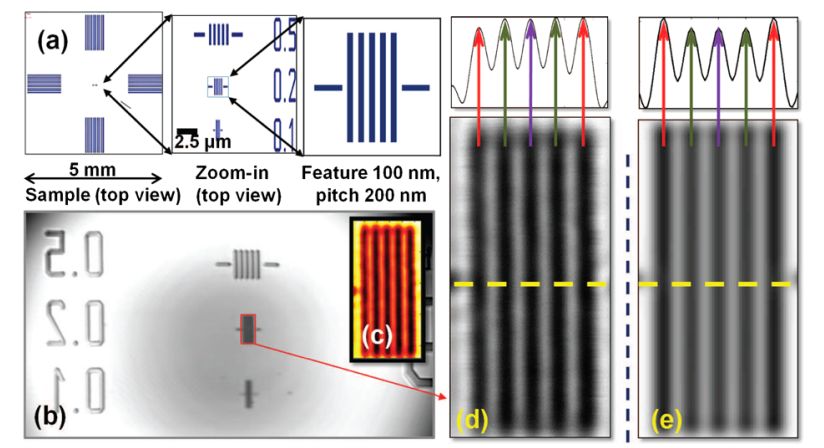

FIG. 2. Our result showing a resolution of 100-nm half-pitch feature. The image obtained using our system for the Tedpella Inc.'s critical dimension calibration or resolution target (a) is shown in (b). The zoom-in of the image of the second feature with $100-\mathrm{nm}$ half pitch is shown in (d). An artificially colorized image is included in (c) for convenient visualization. A simulated image [15] of the feature is shown in (e). The normalized intensity (inverted) at the cross-section lines shown in (d) and (e) are plotted above them. 


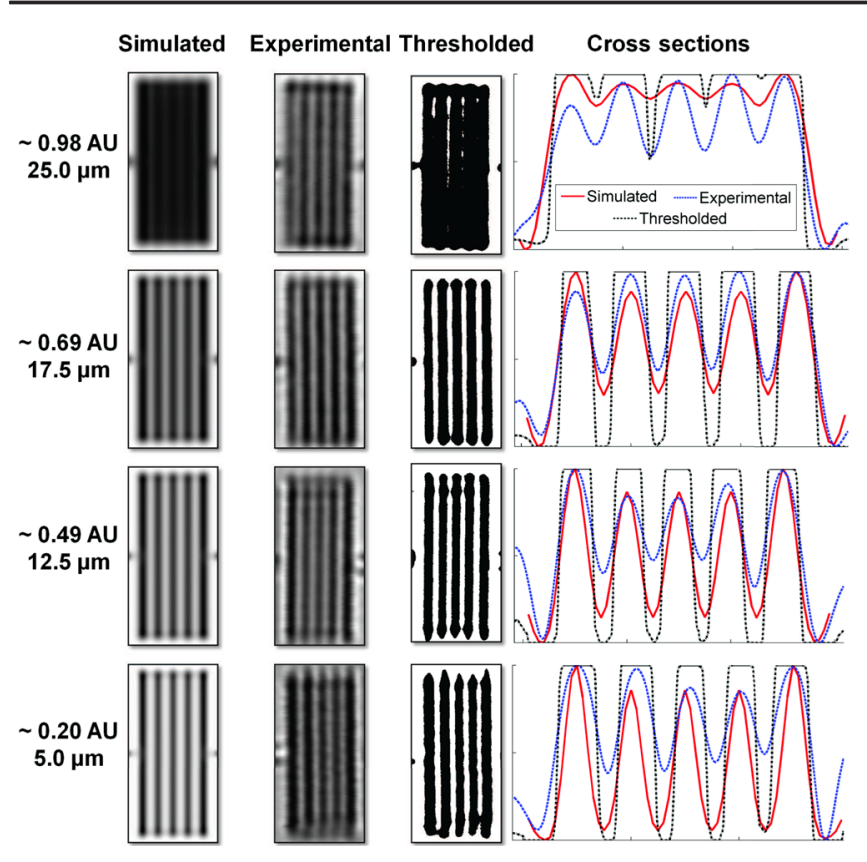

FIG. 3. Images using different pinhole radii. This figure shows the simulated and experimental images obtained using different pinhole radii. Binary images obtained using Otsu's thresholding method as shown in Fig. 4(a) are also shown. The cross-section curves for simulated, experimental, and thresholded images are also shown. The cross-section curves for experimental and thresholded images are obtained using the processing shown in Fig. 4(a).

can be distinctly identified. Thus, it is evident that we have achieved an experimental resolution of $100 \mathrm{~nm}(0.094 \lambda)$ using a wavelength of $1064 \mathrm{~nm}$ and a NIR-ASIL system with N.A.SIL $=3.3$. We also compare this image with the simulated image of this feature and system configuration, which is shown in Fig. 2(e). The intensity at the cross section is also shown. It is seen that the experimental result matches quite well with the simulated image.

For further assessment, we image the feature using different practically available pinhole radii and show qualitative and quantitative comparison in Fig. 3 and (a)

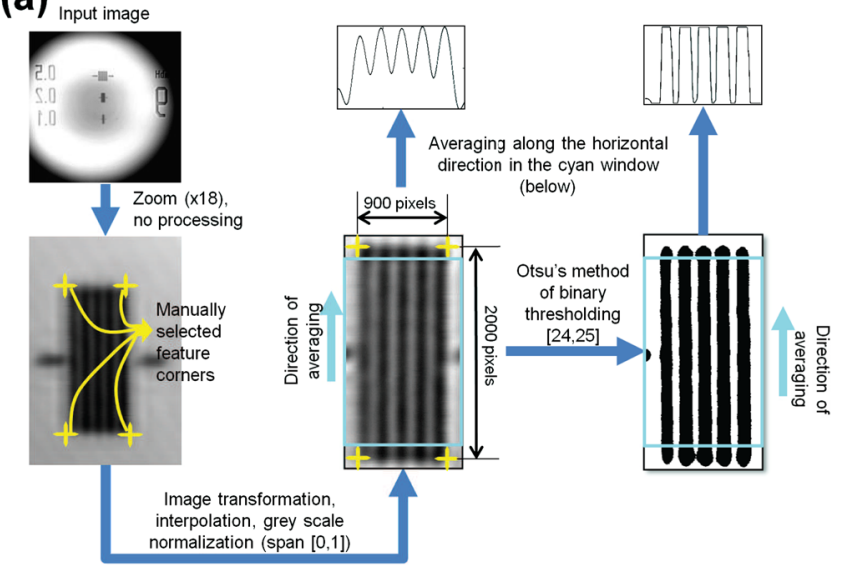

(b)

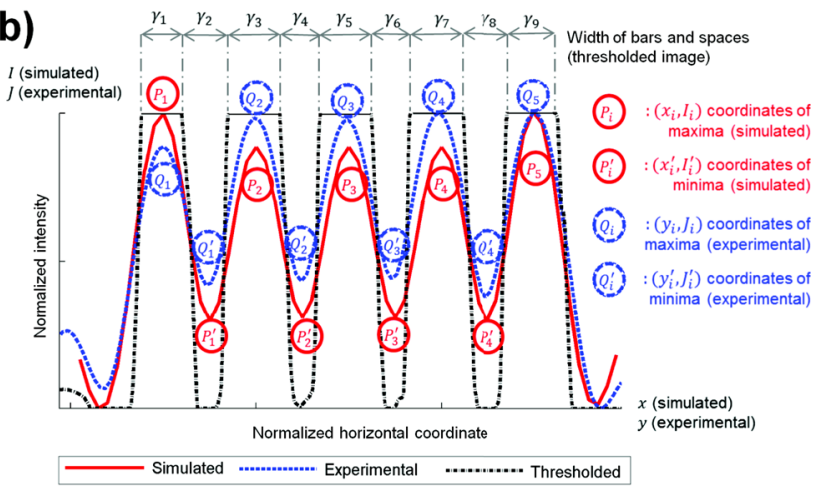

FIG. 4. Binary thresholded image and cross-section curves, and their notations. Panel (a) shows the image processing steps done to obtain the binary thresholded image and the cross-section curves for the experimental and the thresholded images. Panel (b) shows the notations used in (a) for deriving quantitative metrics of image quality of the feature.

Table I, respectively. The quantities used in Table I and the processing we use for obtaining the cross-section curves are explained using Fig. 4. Further discussion on the quantitative formulas is given in Sec. III C. We also show binary images obtained by gray-level thresholding in Fig. 3, where Otsu's method of threshold detection from gray-level

TABLE I. Quantitative assessment of images with different pinhole radii.

\begin{tabular}{|c|c|c|c|c|c|c|c|}
\hline \multirow{2}{*}{$\begin{array}{l}\text { Radius of pinhole } \\
(\mathrm{AU})\end{array}$} & \multicolumn{2}{|c|}{ In-feature contrast } & \multicolumn{2}{|c|}{ Feature contrast } & \multicolumn{3}{|c|}{ Nonuniformity } \\
\hline & Simulated $^{\text {(a) }}$ & Experimental $^{(b)}$ & Simulated $^{(\mathrm{c})}$ & Experimental $^{(\mathrm{d})}$ & $\operatorname{Maxima}^{(\mathrm{e})}$ & Minima $^{(\mathrm{f})}$ & widths $^{(\mathrm{g})}$ \\
\hline 0.98 & 0.0581 & 0.3317 & 0.9303 & 0.7157 & 0.0453 & 0.0388 & 1.2857 \\
\hline 0.69 & 0.7560 & 0.7337 & 0.6720 & 0.6675 & 0.0552 & 0.0547 & 0.2910 \\
\hline 0.49 & 1.2398 & 0.6267 & 0.5548 & 0.6527 & 0.1097 & 0.0653 & 0.3185 \\
\hline 0.20 & 1.7860 & 0.5833 & 0.4684 & 0.6717 & 0.1510 & 0.1440 & 0.6758 \\
\hline Formulas (see Fig. & for notations) & & & & & & \\
\hline
\end{tabular}

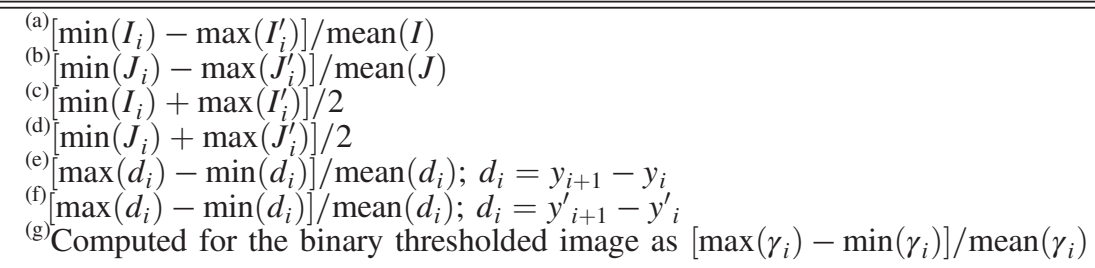




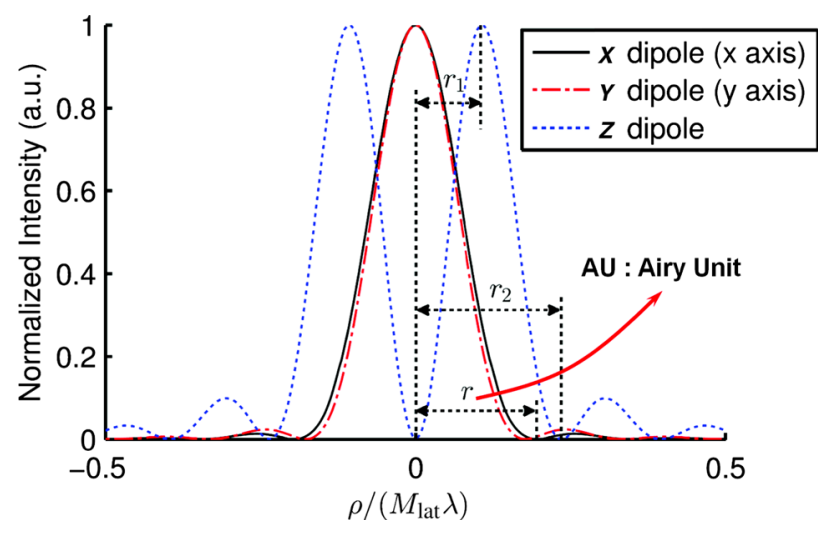

FIG. 5. Point-spread function and candidates for pinhole radii. The cross section of images (point-spread function) of $x, y, z$ dipoles at the focal point are plotted here. $x-y$ plane is the lateral plane and $z$ axis is the longitudinal direction. One Airy unit (AU) given by $r$ (here, $r=25.4 \mu \mathrm{m}$ ) corresponds to the first zero of the point-spread function of $x$ dipole. Dimensions $r_{1}=13.7 \mu \mathrm{m}$ and $r_{2}=30.6 \mu \mathrm{m}$ correspond to the peak and the first zero after the peak of the point-spread function of the $z$ dipole, respectively.

histograms is used [24,25]. Further, the point-spread function (PSF) and the Airy-disk radius (referred to as one Airy unit AU) used in Fig. 3 are shown in Fig. 5. The Airy-disk radius corresponding to the first zero of the PSF of the $x$ dipole is $r=25.4 \mu \mathrm{m}$. For completeness, we also note the peak and first zero of the PSF of the $z$ dipole, $r_{1}=13.7 \mu \mathrm{m}$ and $r_{2}=30.6 \mu \mathrm{m}$, respectively.

The pinholes considered in this paper have radii $(25,17.5,12.5,5) \mu \mathrm{m}$. These values correspond to approximately $0.98,0.69,0.49$, and $0.2 \mathrm{AU}$, respectively. We show in Fig. 3 that pinholes of 0.69 and 0.49 AU provide the best images. Among these two, the experimental image of the $0.69 \mathrm{AU}$ has a better contrast and a better match with the simulated results (see Sec. III B for more details). Also, the results corresponding to these pinhole radii clearly demonstrate improvement over the existing benchmark [6].

In Table I, we list the in-feature and the feature contrasts for the simulated and experimental cross sections. They are defined in formulas (a)-(d) in Table I. We note that the infeature contrast here is the contrast between the details of the feature itself while the feature contrast is the contrast of the overall feature with the image background. Ideally, it is desirable to have a balance of both quantities. This is because in-feature contrast helps in resolving the details of the feature while the feature contrast ensures that the signalto-noise ratio over the feature is larger than the background, and thus the image of the feature is less sensitive to the system noise. Accordingly, we see from the simulated infeature and feature contrasts that $0.69 \mathrm{AU}$ provides the best choice among the four pinhole radii. We note that even the experimental in-feature and feature contrast for $0.69 \mathrm{AU}$ provides a good match with the simulated values.

It is interesting to note that the experimental in-feature and feature contrasts for other pinhole radii are different from the simulated values. This is because the brightness and contrast of our system is electronically chosen, with a very small nonlinearity, to allow for visually best capturing of the background as well as the feature. This, in fact, helps in improving the in-feature contrast for the image with $0.98 \mathrm{AU}$ pinhole radius and the feature contrasts for 0.49 and $0.2 \mathrm{AU}$ radii pinholes.

The other quantity that is shown in Table $I$ is the nonuniformity of the maxima, minima, and the bar widths computed from the binary image. The feature considered in the paper has uniform widths of bars and spaces. Thus, it is expected that the maxima and minima are also uniformly spaced. To quantify this aspect, we use the formulas (e) and (f) in Table I. The quantities denote the nonuniformity since they measure the difference in distances between consecutive maxima (or minima) with respect to the average distance between them. Thus, the lower the value of these metrics, the more uniform the feature geometry appears in the image. It is seen that the image with $0.98 \mathrm{AU}$ pinhole radius has the minimum nonuniformity, and it is closely followed by $0.69 \mathrm{AU}$ pinhole radius. Further, in the thresholded binary image, we find the widths of the bars and spaces in the cross section ( $\gamma_{1}$ to $\gamma_{9}$ ) shown in Fig. 4(b). Then, the nonuniformity of the widths of the bars and spaces is computed using formula $(\mathrm{g})$ in Table I. For this measure, the image with 0.98 AU fails miserably, mainly because the bars in the corresponding image are not clearly separable and the spaces are very small, as seen in Fig. 3. In this respect, the image with $0.69 \mathrm{AU}$ performs the best and gives good uniformity of the bar and space widths. All of the above results clearly indicate that the best imaging solution is provided by the pinhole with radius $0.69 \mathrm{AU}$.

\section{DISCUSSION}

\section{A. Focusing through ASIL}

We first discuss focusing in ASIL briefly. If a sphere of radius $R$ with refractive index $n$ (silicon) is placed in a focusing beam such that the distance between the center of the sphere and the focal point of the focusing beam is $n R$, then the beam focuses inside the sphere at a distance $R / n$, as shown in Fig. 6(a). Such focusing has several salient features, which include (1) an aberration-free focal spot, (2) an enhanced effective numerical aperture N.A.SIL $=$ $n^{2} \mathrm{~N}$.A.focus, where N.A.focus is the numerical aperture of the focusing beam and the condition of total internal reflection is not encountered, and (3) a lateral magnification of $n^{2}$ and a longitudinal magnification of $n^{3}$. The benefits of the ASIL are slightly traded off because the depth of focus of the ASIL system is very small [16], and the ASIL system is prone to aberration $[8,11,20-22]$ in nonideal measurement conditions. There are two main sources of aberration. The first source is the inherent geometric aberration of the ASIL system at planes away from the ASIL's focal plane. This aberration can be avoided by precisely imaging 

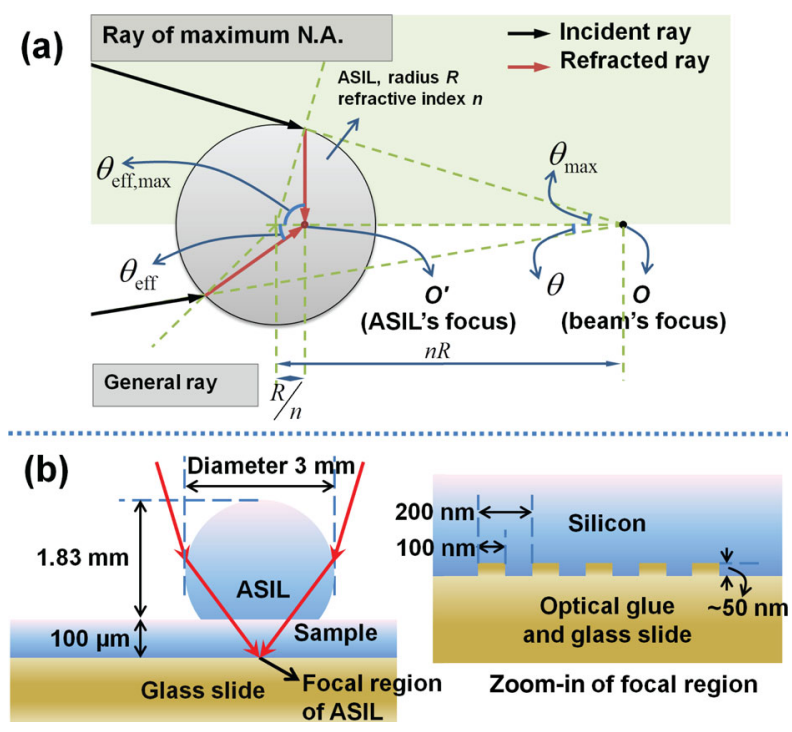

FIG. 6. Focusing through ASIL and geometry of ASIL and sample. The geometric configuration of ASIL, location of its focal point, and refraction at ASIL interface is shown in (a). Geometric details of ASIL used in our experiment and a sample prepared for imaging are shown in (b).

at the focal plane of the sample. The second source is the discontinuity at the ASIL and sample interface. The refraction of the beam at the air gap destroys the aberration-free property of ASIL's focal spot. Both of these issues are dealt with in our paper using a patented SIL assembly discussed in Sec. IV.

\section{B. Simulation of images for our system}

We highlight that our imaging system is a high N.A. (N.A.SIL $=3.3$ ) coherent scanning mode imaging microscope. Thus, low N.A. or paraxial approximations do not apply to our system, and knowledge of the PSF alone is not sufficient for simulating or interpreting the images and optimizing the imaging system. Instead, complete 3D simulation of vectorial electric fields, i.e., solving Maxwell's equations, is required for correctly understanding the system. In simulation, we assume that the optical glue (refractive index same as silica) fills the feature cavities [see Fig. 6(b), right-hand side)], though practically some of the cavities may remain empty or only partially filled. We use the computational model of Ref. [15]. Corresponding to Ref. [15], we consider 200 discrete Fourier and chirp $\mathrm{Z}$ transform components, where the focal region is a circular disk with radius $1600 \mathrm{~nm}$ $(\approx 1.5 \lambda)$ and longitudinal width of $20 \mathrm{~nm}$. Each voxel in the focal region is a cube of size $20 \mathrm{~nm}$. In the imaging plane, before integrating over the pinhole's aperture, the computation grid is the exact analog of the focal region. After integration of the intensity over the pinhole's aperture, the intensity for each pixel is obtained. Finally, pixels in the imaging plane due to scanning also correspond to $20 \mathrm{~nm}$ in the focal plane.

\section{Resolution for our feature}

Since our feature is an extended nonpointlike feature, we cannot use the conventional two-point resolution criteria, such as the Sparrow criterion [26], for obtaining the resolution limit of the system. Yet, it is interesting to consider how the observed resolution of $100 \mathrm{~nm}$ (for our extended feature) compares with the Sparrow resolution limit. The Sparrow resolution limit computed for a nonscanning ASIL system of N.A.sIL $=3.3$ using the PSF of the collection path is $\approx \lambda / 4$, as reported in Ref. [7]. In this sense, the observed resolution of $100 \mathrm{~nm}$ using 1064-nm wavelength (i.e., $\lambda / 10.6$ ) in Fig. 2 is significantly better. However, we note that the Sparrow resolution limit does not apply to our system even for the prediction of two-point resolution since the resolution of our system is influenced by the focusing PSF and is further improved by the scanning mode, as noted in Ref. [15].

As a consequence of the above limits, the resolution for an extended feature and a system such as ours needs to be defined differently. According to Smith [27], the only resolution criterion is, "can we discern the lines?," and that all the coarser features as well as the number of lines are discernible. The use of contrast and other such measures are considered as qualitative and susceptible to individual interpretation, and thus discouraged. Nevertheless, quantitative measures that address the criteria of Smith [27] can be designed, as we have done here. Here, we define resolution indirectly using the in-feature contrast (discerning the lines), feature contrast (discerning the coarser features), and bar and space width nonuniformity (discerning the lines after automatic threshold), as shown in Table I. If the in-feature and feature contrast are sufficiently high, say 0.5 , and bar and space width nonuniformity is sufficiently small, say 0.3 , we can consider the feature as resolved. Then, according to Table I, only a pinhole of $0.69 \mathrm{AU}$ is able to resolve the feature. For a different feature, a suitable quantitative measure of resolution will need to consider the geometry of that specific feature.

\section{Role of pinhole radius}

Now, we discuss the result reported in Fig. 3. We note that the improvement in resolution as compared to the previous benchmark [6] results from the suitable choice of the pinhole radius. As compared to the conventionally used pinhole with a radius of $1 \mathrm{AU}(25.4 \mu \mathrm{m})$, we also consider smaller pinholes $(0.69,0.49,0.2 \mathrm{AU})$. At the same time, the pinholes are large enough not to qualify as a true confocal microscope. The choice of an appropriate pinhole is crucial since the ASIL microscope with N.A.SIL $=3.3$ is a high numerical aperture system.

We note that while longitudinal currents are often small in low N.A. microscopy systems, they are comparable to the lateral currents in high N.A. microscopy systems and play an important role in determining the image quality, as we discuss next. The image formation of NIR-ASIL 
microscopy can be explained using radiation from currents induced on the features due to the focal electric field $[14,15]$.

The different radii of the pinhole allow different proportions of intensities from the lateral and longitudinal current distributions in the focal region of ASIL. The pointspread function for infinitesimal $x, y, z$ directed induced dipoles at the focal point is shown in Fig. 5. Here, the $x-y$ plane is the lateral or transverse plane and the $z$ axis is the longitudinal direction. It is seen that the image of a longitudinal ( $z$ ) current dipole is shaped like a doughnut, as opposed to the spotlike image of lateral $x$ and $y$ dipoles. The field due to the longitudinal current distribution can constructively or destructively interfere with the field due to the lateral current distribution and modify the intensity pattern in the detector region [28]. For example, we show the lateral and longitudinal currents and their contribution in the detector intensity for three scanning points (two on the bars in the feature and one on the space between the two bars) in Fig. 7. The field due to longitudinal currents destructively interferes with the field due to lateral currents for all three points, as seen in the right-hand panel of Fig. 7. The actual intensity pattern (after the interference) for the first and third scanning points (both on the bars) is still a spot. Interestingly, for the second scanning point (on the space), though the pattern for each component is spotlike, the intensity pattern after interference is doughnut shaped.

Large pinhole radii such as $25 \mu \mathrm{m}$ add the intensity from the doughnut portion as well. On the other hand, small pinhole radii such as 12.5 and $5 \mu \mathrm{m}$ avoid collecting light from the doughnut-shaped portion for the second scanning point. Thus, they provide a better contrast between the bars and the space (i.e., in-feature contrast). Since the smaller pinholes collect only a small amount of intensity from the spot when the scanning point is on the bar (the first and the third scanning points) and almost zero intensity from the center of the doughnut when the scanning point is in the space (the second scanning point), their feature contrast (between the overall feature and the background) is low. On the other hand, pinhole radius of $17.5 \mu \mathrm{m}$ collects most intensity from the spot for the first and the third scanning point and only a small amount of intensity from the doughnut for the second scanning point, thus giving good in-feature contrast as well as feature contrast. As a consequence of good feature contrast, the image of the feature is expected to be less sensitive to the system noise as well, since the signal-to-noise ratio over the feature is larger in this case.

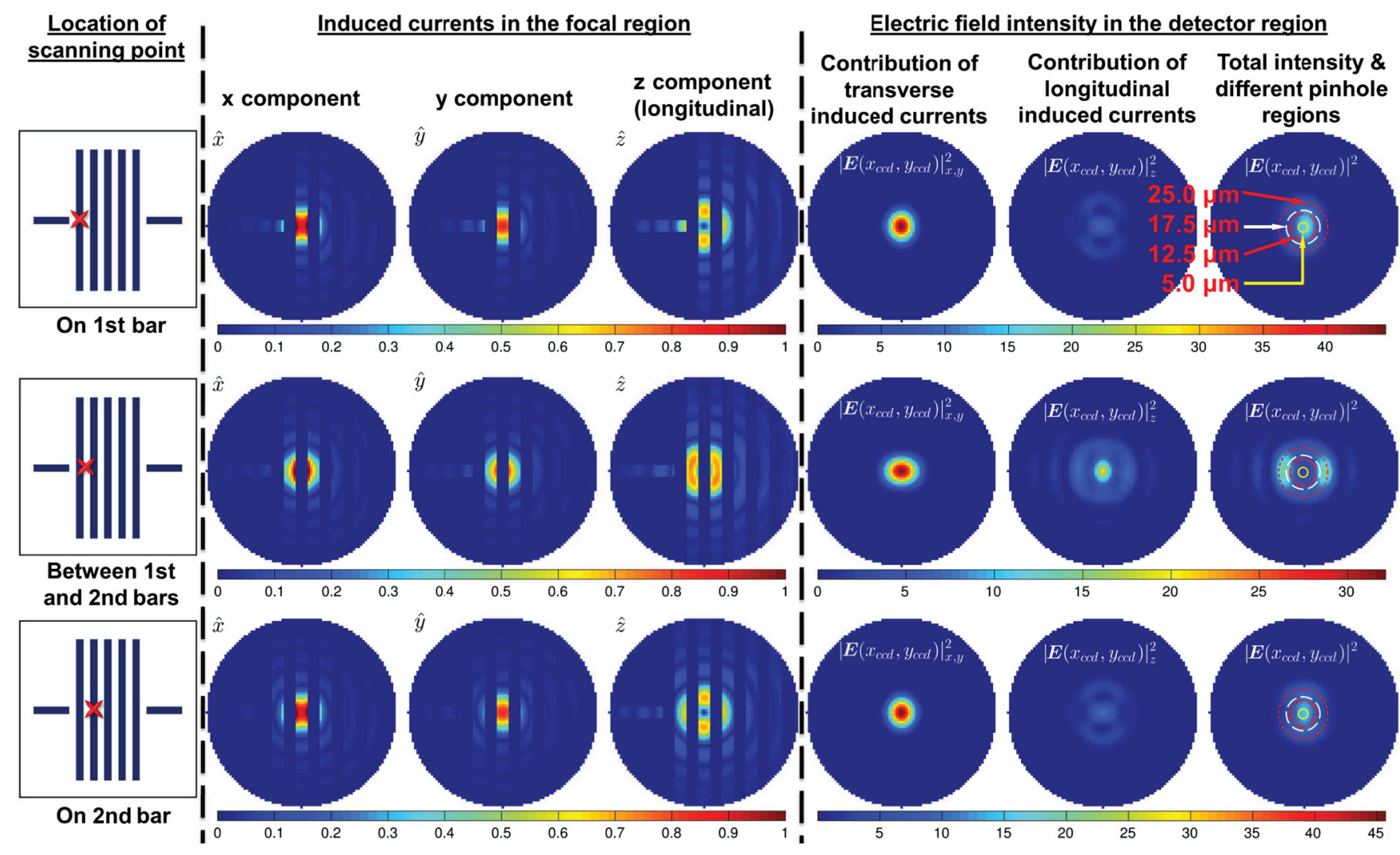

FIG. 7. Effect of longitudinal currents and pinhole size. Analysis of image formation and contribution of longitudinal currents for three scanning points. Three scanning points, shown in the left-hand panel, are considered. Induced current distributions in the focal plane (center panel) and electrical intensities at the detector (right-hand panel) corresponding to each scanning point are shown adjacent to it. The $x-y$ plane is the lateral or transverse plane and the $z$ axis is the longitudinal direction. In the rightmost figures, along with the total electric intensity at the detector, the integration regions for various pinholes are also shown. 


\section{E. Future of this technology}

Since this technology can satisfy the required resolution limit for the semiconductor industry [1], it will directly help in yield enhancement in the semiconductor industry and shorten the automatic test time cycles significantly. Further, this technology has scope for significant resolution enhancement so that it can cater to coming technologies as well. A proof of concept is shown in Ref. [29], where it is proposed to design filters for ASIL microscopy that are specifically suitable for imaging features of a known geometry and material. It is seen that a simple two-layer binary phase filter can be used in the focusing path to image three-bar features of half-pitch $\lambda / 12.5$, about $85 \mathrm{~nm}$ for $\lambda=1064 \mathrm{~nm}$. Indeed, more sophisticated and complex filters and filter placement strategies can cater to complicated features with smaller half pitch. This approach is quite suitable for semiconductor technology since the transistors are standard features repeated throughout the wafer. Thus, this technology has sufficient scope of improvement and evolution so that it can cater to the semiconductor industry's demands for at least a few more decades. Further, we note that our study shows the importance of a suitable pinhole size in high N.A. imaging systems, such as ASIL microscopy here. We expect that this work should influence resolution improvement of other high N.A. imaging systems as well, such as in biotechnology and material nanoimaging applications.

\section{METHODS}

\section{A. Microscope}

The scanning optical microscopy system was assembled in-house on an optical table. All of the wideband optics are antireflection coated. In order to use the entire N.A.SIL $=$ 3.3 available using the SIL assembly [19], which corresponds to a focusing beam N.A. of 0.27 , we use a NIR objective with N.A. 0.4 .

\section{B. SIL assembly}

A patented assembly [19] designed specifically for the purpose of holding and accurately aligning the ASIL, pressing it onto the sample using a mechanical spring system to avoid an air gap between ASIL and the sample, and finding the correct focal plane enables the mitigation of the occurrence and effect of aberration in imaging using ASIL. This assembly provides an effective numerical aperture N.A.SIL of 3.3 and requires ASIL of diameter $3 \mathrm{~mm}$. Further, the assembly requires the height of ASIL to be $1.83 \pm 0.005 \mathrm{~mm}$.

\section{Sample preparation}

The top surface of the sample (containing the features) is glued onto a glass slide using UV curing optical glue with the same refractive index as the glass slide. As discussed above, the assembly requires the height of ASIL to be $1.83 \pm 0.005 \mathrm{~mm}$, whereas the value of $R / n$ for such ASIL is $0.429 \mathrm{~mm}$. On the other hand, the height of the substrate of TedPella Inc.'s critical dimension calibration or resolution test target, used as the sample, is $750 \mu \mathrm{m}$, with features etched upon its top surface. Therefore, the sample is polished such that the height between the features and the ASIL-sample interface is about $100 \mu \mathrm{m}$. See Fig. 6(b) for illustration.

\section{ACKNOWLEDGMENTS}

This research is supported by the National Research Foundation, Prime Ministers Office, Singapore under its Competitive Research Programme (CRP Award No. NRFCRP10-2012-04) and Singapore's Ministry of Education (Grant No. MOE2009-T2-2-086). Semicaps Pte. Ltd. provided their proprietary scanning system and ASIL assembly on loan.

[1] Yield Enhancement, Technical Report, International Technology Roadmap for Semiconductors, 2013.

[2] S. M. Mansfield and G. S. Kino, Solid Immersion Microscope, Appl. Phys. Lett. 57, 2615 (1990).

[3] A. N. Vamivakas, R. D. Younger, B. B. Goldberg, A. K. Swan, M. S.Ünlü, E. R. Behringer, and S. B. Ippolito, A Case Study for Optics: The Solid Immersion Microscope, Am. J. Phys. 76, 758 (2008).

[4] S. B. Ippolito, B. B. Goldberg, and M. S. Ünlü, High Spatial Resolution Subsurface Microscopy., Appl. Phys. Lett. 78, 4071 (2001).

[5] F. H. Köklü, J. I. Quesnel, A. N. Vamivakas, S. B. Ippolito, B. B. Goldberg, and M.S. Ünlü, Widefield Subsurface Microscopy of Integrated Circuits, Opt. Express 16, 9501 (2008).

[6] Semicaps RSIL-Refractive Solid Immersion Lens, Technical Report, Semicaps Pte. Ltd. Singapore, 2012.

[7] R. Chen, K. Agarwal, C. J. R. Sheppard, J. C. H. Phang, and X. Chen, Resolution of Aplanatic Solid Immersion Lens Based Microscopy, J. Opt. Soc. Am. A 29, 1059 (2012).

[8] S. H. Goh and C. J. R. Sheppard, High Aperture Focusing through a Spherical Interface: Application to Refractive Solid Immersion Lens (RSIL) for Subsurface Imaging, Opt. Commun. 282, 1036 (2009).

[9] L. E. Helseth, Roles of Polarization, Phase and Amplitude in Solid Immersion Lens Systems, Opt. Commun. 191, 161 (2001).

[10] T. X. Hoang, X. Chen, and C. J. R. Sheppard, Multipole Theory for Tight Focusing of Polarized Light, Including Radially Polarized and Other Special Cases, J. Opt. Soc. Am. A 29, 32 (2012).

[11] K. M. Lim, G. C. F. Lee, C. J. R. Sheppard, J. C. H. Phang, C. L. Wong, and X. Chen, Effect of Polarization on a Solid Immersion Lens of Arbitrary Thickness, J. Opt. Soc. Am. A 28, 903 (2011). 
[12] K. Huang and Y. Li, Realization of a Subwavelength Focused Spot without a Longitudinal Field Component in a Solid Immersion Lens-Based System, Opt. Lett. 36, 3536 (2011).

[13] R. Chen, K. Agarwal, C. J. R. Sheppard, and X. Chen, Imaging Using Cylindrical Vector Beams in a HighNumerical-Aperture Microscopy System, Opt. Lett. 38, 3111 (2013).

[14] R. Chen, K. Agarwal, Y. Zhong, C. J. R. Sheppard, J. C. H. Phang, and X. Chen, Complete Modeling of Subsurface Microscopy System Based on Aplanatic Solid Immersion Lens, J. Opt. Soc. Am. A 29, 2350 (2012).

[15] R. Chen, K. Agarwal, C. J. R. Sheppard, J. C. H. Phang, and X. Chen, A Complete and Computationally Efficient Numerical Model of Aplanatic Solid Immersion Lens Scanning Microscope, Opt. Express 21, 14316 (2013).

[16] L. Hu, R. Chen, K. Agarwal, C. J. R. Sheppard, J. C. H. Phang, and X. Chen, Dyadic Green's Function for Aplanatic Solid Immersion Lens Based Sub-Surface Microscopy, Opt. Express 19, 19280 (2011).

[17] A. Yurt, A. Uyar, T. B. Cilingiroglu, B. B. Goldberg, and M. S. Ünlü, Evanescent Waves in High Numerical Aperture Aplanatic Solid Immersion Microscopy: Effects of Forbidden Light on Subsurface Imaging, Opt. Express 22, 7422 (2014).

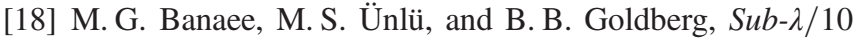
Spot Size in Semiconductor Solid Immersion Lens Microscopy, Opt. Commun. 315, 108 (2014).

[19] C. M. Chua, W. P. Chua, L. S. Koh, C. H. J. Phang, and S. H. Tan, U.S. Patent No. 8,072,699 (2011).
[20] B. B. Goldberg, A. Yurt, Y. Lu, E. Ramsay, F. H. Köklü, J. Mertz, T. G. Bifano, and M.S. Ünlü, Chromatic and Spherical Aberration Correction for Silicon Aplanatic Solid Immersion Lens for Fault Isolation and Photon Emission Microscopy of Integrated Circuits, Microelectron. Reliab. 51, 1637 (2011).

[21] Y. Lu, E. Ramsay, C. R. Stockbridge, A. Yurt, F. H. Köklü, T. G. Bifano, M. S. Ünlü, and B. B. Goldberg, Spherical Aberration Correction in Aplanatic Solid Immersion Lens Imaging Using a MEMS Deformable Mirror, Microelectron. Reliab. 52, 2120 (2012).

[22] Y. Lu, T. G. Bifano, M. S. Ünlü, and B. B. Goldberg, Aberration Compensation in Aplanatic Solid Immersion Lens Microscopy, Opt. Express 21, 28189 (2013).

[23] TedPella Inc., http://www.tedpella.com/metrochip_html/ metrochip-calibration-target.htm.

[24] D. K. Prasad and M. S. Brown, Online Tracking of Deformable Objects under Occlusion Using Dominant Points, J. Opt. Soc. Am. A 30, 1484 (2013).

[25] D. K. Prasad, Fabrication Imperfection Analysis and Statistics Generation Using Precision and Reliability Optimization Method, Opt. Express 21, 17602 (2013).

[26] C.M. Sparrow, On Spectroscopic Resolving Power, Astrophys. J. 44, 76 (1916).

[27] W. J. Smith, Modern Optical Engineering, 3rd ed. (McGraw-Hill, New York, 2000), Chap. 15, p. 593.

[28] R. Chen, Ph.D. thesis, National University of Singapore, 2013.

[29] E. L. Loh, R. Chen, K. Agarwal, and X. Chen, Feature-Based Filter Design for Resolution Enhancement of Known Features in Microscopy, J. Opt. Soc. Am. A 31, 2610 (2014). 